Tetrahedron

journal homepage: www.elsevier.com

\title{
A vesicular self-assembled amphiphilic palladium NNC-pincer complex-catalyzed allylic arylation of allyl acetates with sodium tetraarylborates in water
}

\author{
Go Hamasaka $^{\mathrm{a}, \mathrm{b}}$, Fumie Sakurai ${ }^{\mathrm{a}, \mathrm{b}}$ and Yasuhiro Uozumi ${ }^{\mathrm{a}, \mathrm{b}, \mathrm{c}, \mathrm{d}}{ }_{*}$ \\ ${ }^{a}$ Institute for Molecular Science, Myodaiji, Okazaki 444-8787, Japan \\ ${ }^{b}$ The Graduate University for Advanced Studies, Myodaiji, Okazaki 444-8787, Japan \\ ${ }^{c}$ Green Nanocatalysis Research Team, RIKEN Center for Sustainable Resource Science, Hirosawa, Wako 351-0198, Japan \\ ${ }^{d} J S T$-CREST and JST-ACCEL, Myodaiji, Okazaki 444-8787, Japan
}

\section{ARTICLE INFO}

\section{ABSTRACT}

\section{Article history:}

Received

Received in revised form

Accepted

Available online

\section{Keywords:}

Allylic arylation

Vesicle

Palladium

Pincer complex

Catalysis

Aqueous system

\section{Introduction}

The development of supramolecular catalyst systems as enzyme-inspired systems for the efficient catalysis of various reactions has received considerable attention from many chemists. ${ }^{1}$ In this context, nanosized self-assembled architecturebased catalytic systems, such as bilayer vesicles, have been investigated. ${ }^{2}$ Several research groups have reported that amphiphilic polymers and low-molecular weight amphiphiles containing a catalytically active site can self-assemble to form catalytically active self-assembled nanoarchitectures (micelles, vesicles, nanotubes, etc.) that can be used to induce organic reactions in water. ${ }^{3}$ We recently reported that vesicular selfassembled amphiphilic pincer palladium complexes can catalyze organic transformations in water. ${ }^{4,5}$ For example, amphiphilic palladium NNC-pincer complexes $\mathbf{1 a}$ and $\mathbf{1 b}$ self-assembled in water to form the vesicles $\mathbf{1} \mathbf{a}_{v s c l}$ and $\mathbf{1 b}_{v s c l}$, respectively (Figure 1). Vesicles $\mathbf{1} \mathbf{b}_{\text {vscl }}$ efficiently catalyzed a copper-free Sonogashira coupling reaction in water, whereas the amorphous complex $\mathbf{1} \mathbf{b}_{\text {amps }}$ did not significantly promote the reaction. ${ }^{5} \mathrm{We}$ also showed that the formation of vesicles was necessary for the efficient promotion of the reactions in water. The effect of the formation of vesicular structures is explained as follows (Figure Z). The organic substrates spontaneously concentrate within the hydrophobic region of the membranes of vesicles as a result of hydrophobic interactions. Subsequently, the substrates approach the catalytic center and the organic transformation takes place rapidly as a result of the presence of high concentrations of the substrates near the catalytic center.

The allylic substitution reaction known as the Tsuji-Trost reaction is an important transformation in the synthesis of natural compounds and pharmaceuticals. ${ }^{6}$ In an attempt to demonstrate the scope and utility of our concept (Figure 2), we decided to apply vesicular catalysts to the allylic arylation of allyl acetates with sodium tetraarylborates in water. ${ }^{7}$ Here, we report the application of self-assembled vesicles $\left(\mathbf{1 a}_{v s c l}\right.$ and $\left.\mathbf{1 b}_{v s c l}\right)$ of amphiphilic palladium NNC-pincer complexes $\mathbf{1 a}$ and $\mathbf{1 b}$ as catalysts in the allylic arylation of allyl acetates with sodium tetraarylborates in water. The formation of a vesicular structure is essential for the promotion of this reaction.

\section{Results and discussion}

We examined the reaction of (2E)-3-biphenyl-4-ylprop-2-en1 -yl acetate (2a) with sodium tetraphenylborate (3a) in the presence of amorphous pincer palladium complexes $\mathbf{1} \mathbf{a}_{\text {amps }}$ and $\mathbf{1 b}_{\text {amps }}$ and their self-assembled vesicular nanocomposites $\mathbf{1} \mathbf{a}_{v s c l}$

* Corresponding author. Tel.: +81-564-59-5570; fax: +81-564-59-5574; e-mail: uo@ims.ac.jp

**The authors would like to dedicate this article to Professor Jiro Tsuji for his peerless contribution to the broad discipline of organic chemistry. 

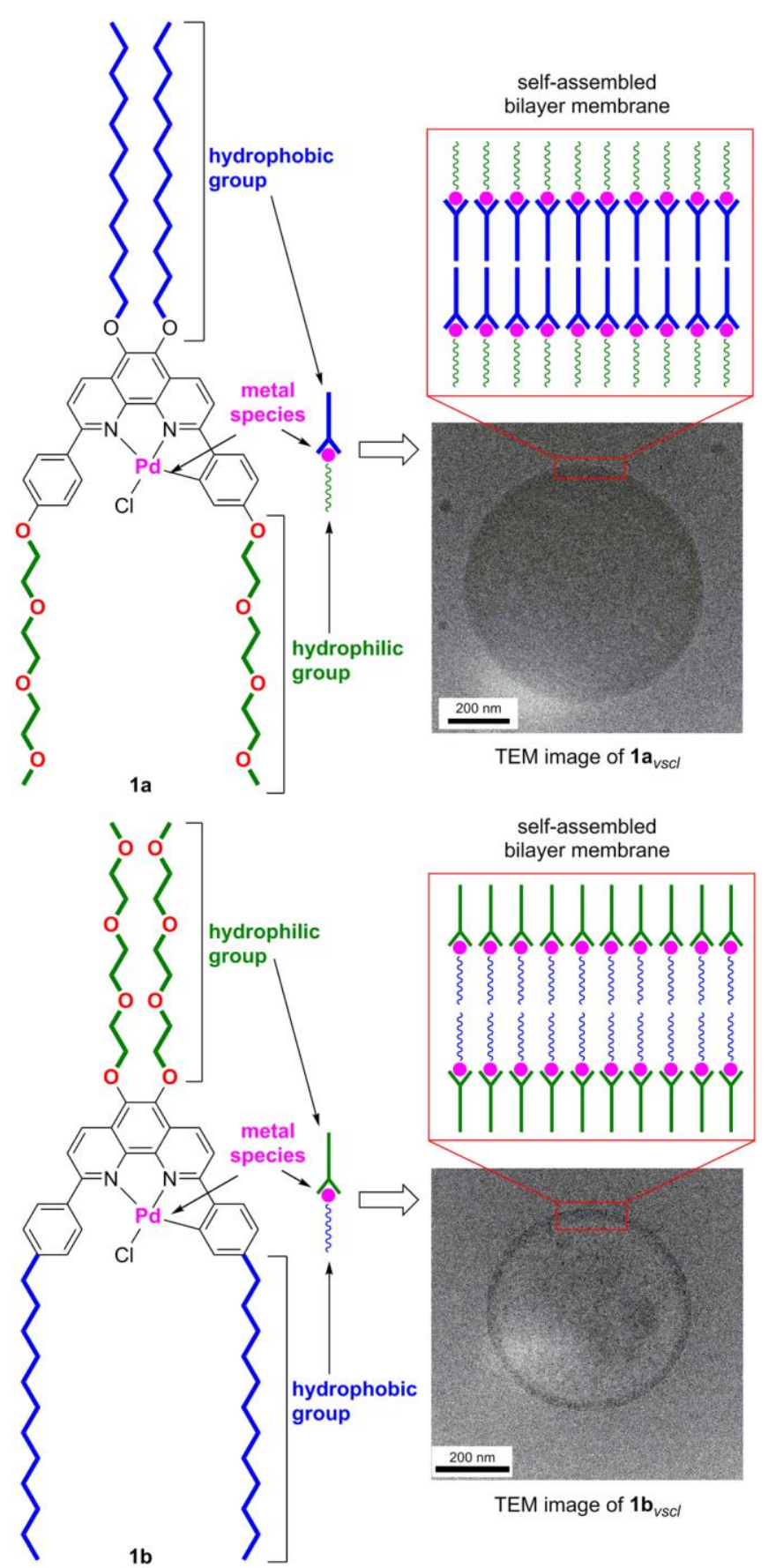

Fig. 1. Self-assembly of amphiphilic pincer Pd complexes $\mathbf{1 a}$ and $\mathbf{1 b}$.

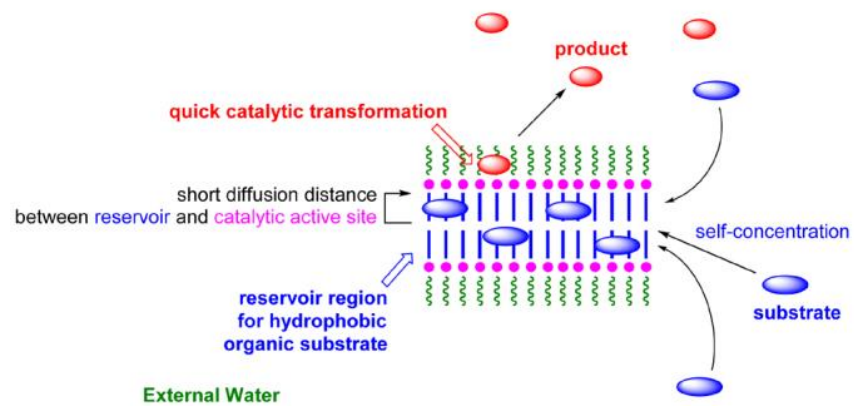

Figure 2. Schematic image of the concept of catalysis within the membranes of vesicles. and $\mathbf{1 b}_{v s c l}$ as catalysts (Table 1). When the reaction was performed with amorphous amphiphilic palladium pincer complex $1 \mathbf{a}_{\text {amps }}$ in water at $50{ }^{\circ} \mathrm{C}$ for $10 \mathrm{~min}$, only an $8 \%$ yield of the desired arylated product 4aa was obtained (entry 1). In contrast, the reaction proceeded smoothly in the presence of the self-assembled vesicular $\mathbf{1 a}_{v s c}$, where the potentially reactive $\mathrm{Pd}-$ $\mathrm{Cl}$ site faced the hydrophilic outer region, as shown in the schematic images of the bilayer membrane of $\mathbf{1 a}_{v s c l}$ [Fig. 1 (top) and Fig. 2], to give 4-[(1E)-3-phenylprop-1-en-1-yl]biphenyl (4aa) in 59\% yield (entry 2). These results show that the formation of vesicles of complex 1a significantly accelerates the allylic arylation reaction in water. The reaction with the amorphous complex $\mathbf{1} \mathbf{b}_{\text {amps }}$ and the corresponding vesicles $\mathbf{1} \mathbf{b}_{\text {vscl }}$ gave $4 \mathbf{a a}$ in 9 and $16 \%$ yield, respectively (entries 3 and 4 ). The formation of a vesicular structure by the self-assembly of $\mathbf{1 b}$ therefore resulted in only a slight promotion of the allylic arylation reaction in water. These results suggested that the position of the hydrophilic and hydrophobic groups on the pincer backbone is critical for efficient promotion of this allylic arylation reaction. When the reaction was carried out in various organic solvents, such as 1,2-dichloroethane (DCE), toluene, acetonitrile, methanol, or $N, N$-dimethylformamide (DMF), the yield of the desired arylated product 4aa was $14 \%$ or less, even when the vesicular composite $\mathbf{1 a}_{\text {vscl }}$ was used as the catalyst (entries 5-16). These results indicated that vesicular $\mathbf{1} \mathbf{a}_{v s c l}$ disassembled or dissolved in organic solvents to give the catalytically less active monomeric 1a. Therefore, the formation of a vesicular architecture was confirmed to be essential for the efficient promotion of the allylic arylation.

Table 1. Effects of catalysts and solvents in the allylic arylation of (2E)-3-biphenyl-4-ylprop-2-en-1-yl acetate (2a) with sodium tetraphenylborate $(\mathbf{3 a})^{\mathrm{a}}$

\begin{tabular}{|c|c|c|c|}
\hline Entry & Catalyst & Solvent & Yield $^{\mathrm{b}}(\%)$ \\
\hline 1 & $\mathbf{1 \mathbf { a } _ { \text { amps } }}$ & $\mathrm{H}_{2} \mathrm{O}$ & 8 \\
\hline 2 & $\mathbf{1} \mathbf{a}_{v s c l}$ & $\mathrm{H}_{2} \mathrm{O}$ & 59 \\
\hline 3 & $1 b_{\text {amps }}$ & $\mathrm{H}_{2} \mathrm{O}$ & 9 \\
\hline 4 & $\mathbf{1} \mathbf{b}_{v s c l}$ & $\mathrm{H}_{2} \mathrm{O}$ & 16 \\
\hline 5 & $\mathbf{1} \mathbf{a}_{a m p s}$ & DCE & 3 \\
\hline 6 & $\mathbf{1} \mathbf{a}_{v s c l}$ & DCE & 8 \\
\hline 7 & $\mathbf{1} \mathbf{a}_{a m p s}$ & toluene & 0 \\
\hline 8 & $\mathbf{1} \mathbf{a}_{v s c l}$ & toluene & 10 \\
\hline 9 & $1 \mathbf{a}_{\text {amps }}$ & THF & 13 \\
\hline 10 & $\mathbf{1} \mathbf{a}_{v s c l}$ & THF & 14 \\
\hline 11 & $\mathbf{1 \mathbf { a } _ { \text { amps } }}$ & $\mathrm{MeCN}$ & 0 \\
\hline 12 & $\mathbf{1 a}_{v s c l}$ & $\mathrm{MeCN}$ & 14 \\
\hline 13 & $\mathbf{1} \mathbf{a}_{a m p s}$ & $\mathrm{MeOH}$ & 14 \\
\hline 14 & $\mathbf{1} \mathbf{a}_{v s c l}$ & $\mathrm{MeOH}$ & 14 \\
\hline 15 & $1 \mathbf{a}_{\text {amps }}$ & DMF & 0 \\
\hline 16 & $\mathbf{1 a}_{v s c l}$ & DMF & 10 \\
\hline
\end{tabular}

Next, we investigated the acceleration effects produced by the formation of the vesicular structure in the allylic arylation of various allyl acetates with sodium tetraarylborates in water 
(Table 2). For all the substrates, the formation of a vesicular structure accelerated the reaction in water. The allylic arylation reaction of cinnamyl acetate $(\mathbf{2 b})$ with sodium tetraphenylborate (3a) in the presence of amorphous complex $\mathbf{1 a}_{\text {amps }}$ gave 1,1'-(1E)prop-1-ene-1,3-diyldibenzene (4ba) in only 9\% yield (entry 1 ). In contrast, the reaction with vesicular $1 \mathbf{a}_{v s c l}$ gave the desired arylated product $\mathbf{4 b a}$ in $82 \%$ yield (entry 2 ). This acceleration effect resulting from the formation of a vesicular structure was also observed in the reaction of sodium tetraphenylborate (3a) with cinnamyl acetates $\mathbf{2 c - 2 e}$, bearing electron-donating or electron-withdrawing substituents (entries 3-8). When the sterically hindered 2-methoxycinnamyl acetate (2f) was subjected to the allylic arylation, the reaction in the presence of catalyst $\mathbf{1}_{v s c l}$ gave 1-methoxy-2-[(1E)-3-phenylprop-1-en-1-yl]benzene (4fa) in $86 \%$ yield (entry 10), whereas catalyst $\mathbf{1} \mathbf{a}_{\text {amps }}$ gave a $29 \%$ yield of $\mathbf{4 f a}$ (entry 9). Vesicles $\mathbf{1} \mathbf{a}_{v s c l}$ also catalyzed the reaction of naphthyl- and thienyl-substituted acetates $\mathbf{2 g}$ and $\mathbf{2 h}$ to give the corresponding arylated products 4ga and 4 ha in 66 and $46 \%$

Table 2. Scope of the allylic arylation of allyl acetates with sodium tetraarylborates ${ }^{\mathrm{a}}$

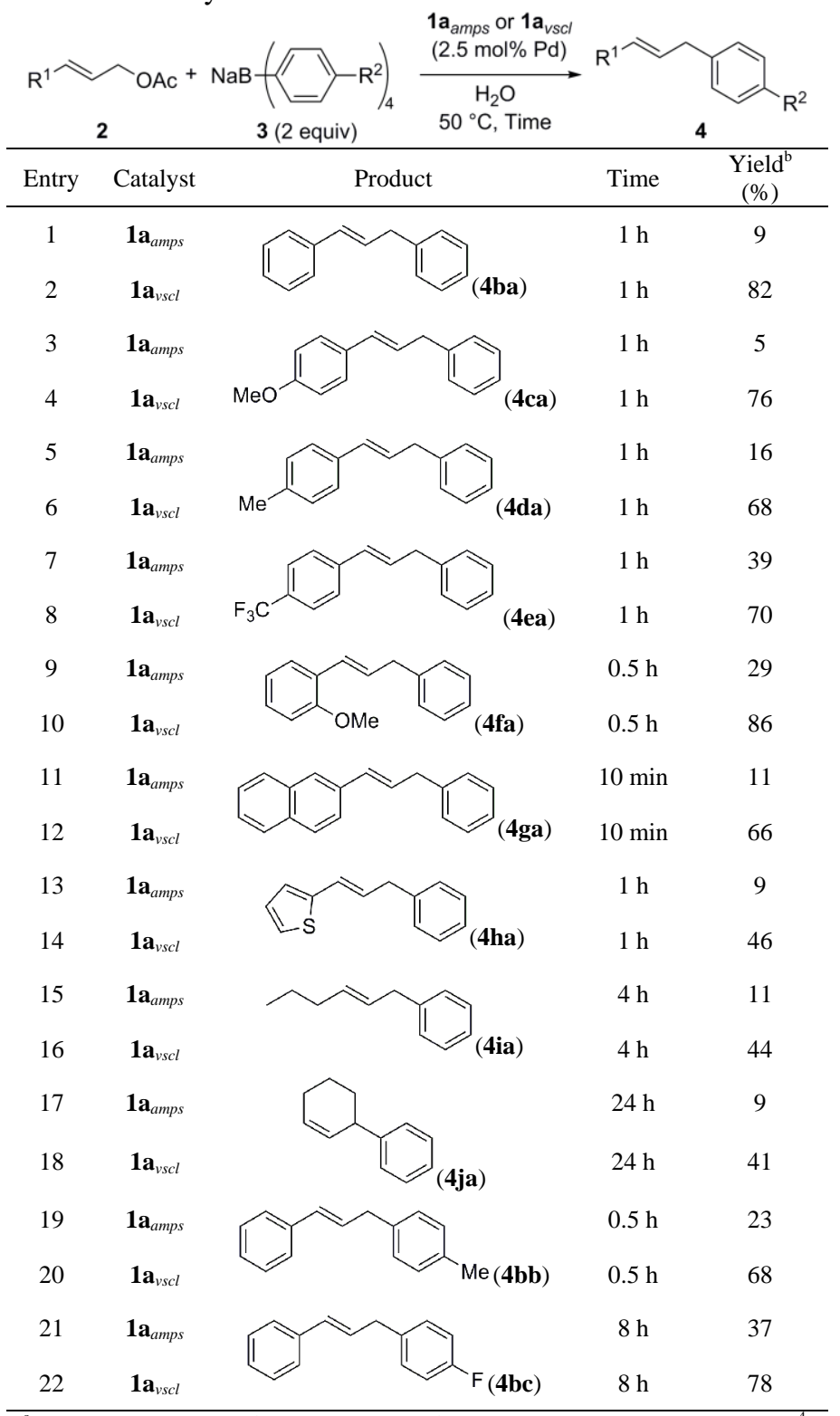

${ }^{\mathrm{a}}$ Reaction conditions: $\mathbf{2 a}(0.034 \mathrm{mmol}), \mathbf{3 a}(0.068 \mathrm{mmol})$, catalyst $\left(8.5 \times 10^{-4}\right.$ $\mathrm{mmol}), \mathrm{H}_{2} \mathrm{O}(1.0 \mathrm{~mL}), 50^{\circ} \mathrm{C}$.

${ }^{\mathrm{b}}$ Isolated yield. yield, respectively (entries 12 and 14). However, the amorphous complex $\mathbf{1} \mathbf{a}_{\text {amps }}$ did not promote the reaction efficiently (entries 11 and 13). We also examined the reaction of less-reactive aliphatic alk-2-enyl acetates with sodium tetraphenylborate (3a). The reactions of hex-2-en-1-yl acetate (2i) and cyclohex-2-en-1yl acetate $(\mathbf{2} \mathbf{j})$ with $\mathbf{3 a}$ in the presence of $\mathbf{1} \mathbf{a}_{\text {amps }}$ proceeded in water to give the corresponding arylated products $\mathbf{4 i a}$ and $\mathbf{4 j a}$ in 11 and $9 \%$ yield, respectively (entries 15 and 17). On the other hand, when vesicular $\mathbf{1} \mathbf{a}_{v s c l}$ was used as the catalyst, the desired products 4ia and 4ja were obtained in 44 and $41 \%$ yield, respectively (entries 16 and 18). A similar acceleration effect resulting from the formation of the vesicular structure was also observed in the reaction of cinnamyl acetate (2b) with sodium tetraarylborates $\mathbf{3 b}$ and $\mathbf{3 c}$ (entries 19-22).

\section{Conclusions}

In summary, vesicles $\mathbf{1} \mathbf{a}_{v s c l}$ showed a superior catalytic activity to that of the amorphous complex $\mathbf{1 a}_{\text {amps }}$ in the allylic arylation reaction of allyl acetates with sodium tetraarylborates in water. The formation of a vesicular structure is essential for efficient promotion of this reaction. On the other hand, when vesicles $\mathbf{1} \mathbf{b}_{v s c l}$ and amorphous complex $\mathbf{1 b}_{\text {amps }}$ were used as catalysts, the formation of vesicles $\mathbf{1 b}_{v s c l}$ did not significantly improve the catalytic activity for the reaction. The directions of the hydrophilic chains and the hydrophobic chains attached to the phenanthroline backbone influence the catalytic activity. Other catalytic applications of the vesicular catalysts $\mathbf{1 a}_{v s c l}$ and $\mathbf{1} \mathbf{b}_{v s c l}$ are currently under investigation in our laboratory.

\section{Experimental section \\ 4.1. General information}

Commercially available chemicals (purchased from SigmaAldrich, TCI, Kanto Chemical, Wako Pure Chemical Industries, Nacalai Tesque, or Merck) were used without further purification unless otherwise noted. Silica gel was purchased from Kanto Chemical (Silica gel 60N; spherical neutral) or Yamazen Corp. (Hi-Flash ${ }^{\mathrm{TM}}$ Column Silica gel). TLC plates were purchased from Merck (TLC Silica gel $60 \mathrm{~F}_{254}$ ). NMR spectra were recorded on a JEOL JNM ECS-400 spectrometer $\left(396 \mathrm{MHz}\right.$ for ${ }^{1} \mathrm{H}, 100 \mathrm{MHz}$ for $\left.{ }^{13} \mathrm{C}\right) .{ }^{1} \mathrm{H}$ NMR chemical shifts are reported in $\delta(\mathrm{ppm})$ relative to tetramethylsilane as internal standard; ${ }^{13} \mathrm{C}$ NMR chemical shifts are relative to $\mathrm{CDCl}_{3}$ as internal standard $(\delta=77.0) .{ }^{1} \mathrm{H}$ and ${ }^{13} \mathrm{C}$ NMR spectra were recorded in $\mathrm{CDCl}_{3}$ at $25^{\circ} \mathrm{C}$. GC/MS analyses were performed with an Agilent 6890 GC/5973N MS Detector. Elemental analyses were performed on a J-Science Lab Micro Corder JM10. Melting points were determined by using a Yanaco MP-J3 micro melting point apparatus and are uncorrected. IR spectra were obtained by using a JASCO FT/IR460plus spectrometer in the ATR mode. Pure water was obtained from a Millipore Milli-Q Academic A10 purification unit. (2E)3-Biphenyl-4-ylprop-2-en-1-yl acetate (2a) ${ }^{8} \quad(2 E)-3-(4-$ methoxyphenyl)prop-2-en-1-yl acetate $\quad(\mathbf{2 c}),{ }^{8} \quad(2 E)-3-(4-$ methylphenyl)prop-2-en-1-yl acetate (2d), ${ }^{8} \quad(2 E)-3-[4-$ (trifluoromethyl)phenyl]prop-2-en-1-yl acetate $(\mathbf{2 e}){ }^{8},{ }^{8}(2 E)-3-(2-$ methoxyphenyl)prop-2-en-1-yl acetate $\quad(\mathbf{2 f}),{ }^{8} \quad(2 E)-3-(2-$ naphthyl)prop-2-en-1-yl acetate (2g) ${ }^{9}$ (2E)-3-(2-thienyl)prop-2en-1-yl acetate $(\mathbf{2 h}),{ }^{8}$ cyclohex-2-en-1-yl acetate $(\mathbf{2} \mathbf{j}),{ }^{10}$ sodium tetrakis(4-methylphenyl)borate $\mathbf{( 3 b}),{ }^{11}$ and sodium tetrakis(4fluorophenyl)borate $(\mathbf{3 c})^{11}$ were prepared by the reported methods.

\subsection{Preparation of $\mathbf{1} \boldsymbol{a}_{v s c l}$}

A chloroform solution of $1 \mathbf{a}(0.1 \mathrm{~mL}, 10 \mathrm{mg} / \mathrm{mL})$ was charged in a $4 \mathrm{~mL}$ vial equipped with a screw cap. After evaporation of 
the chloroform, a thin film of 1a was formed on the inner glass surface of the vial. Then, $1 \mathrm{~mL}$ of Millipore water was added to the vial followed by heating the resulting mixture at $80{ }^{\circ} \mathrm{C}$ for 12 $\mathrm{h}$ without stirring. After standing at $25{ }^{\circ} \mathrm{C}$ for overnight, the resulting mixture was sonicated for $10 \mathrm{~min}$ to generate a yellow suspension of vesicles $\mathbf{1} \mathbf{a}_{\text {vscl }}$.

\subsection{Preparation of $\mathbf{1} \boldsymbol{b}_{v s c l}$}

A chloroform solution of $\mathbf{1 b}(0.1 \mathrm{~mL}, 10 \mathrm{mg} / \mathrm{mL})$ was charged in a $4 \mathrm{~mL}$ vial equipped with a screw cap. After evaporation of the chloroform, a thin film of $\mathbf{1 b}$ was formed on the inner glass surface of the vial. Then, $1 \mathrm{~mL}$ of Millipore water was added to the vial followed by heating the resulting mixture at $60{ }^{\circ} \mathrm{C}$ for $4 \mathrm{~h}$ without stirring. After standing at $25{ }^{\circ} \mathrm{C}$ for overnight, the resulting mixture was sonicated for $10 \mathrm{~min}$ to generate a yellow suspension of vesicles $\mathbf{1} \mathbf{b}_{\text {vscl }}$.

\subsection{Allylic arylation in water; typical procedure}

A VIAL EQUIPPED WITH A SCREW CAP WAS CHARGED WITH A SUSPENSION OF $\boldsymbol{I a}_{v s c l}\left(1.0 \mathrm{MG}, 8.5 \times 10^{-4} \mathrm{MMOL}\right)$, $\mathrm{NABPH}_{4}(3 a)$ (23.3 MG, $\left.0.068 \mathrm{MMOL}\right)$, AND (2E)-3-BIPHENYL4-YLPROP-2-EN-1-YL ACETATE (2a) (8.6 MG, 0.034 MMOL) IN $\mathrm{H}_{2} \mathrm{O}$ (1 ML). THE MIXTURE WAS AGITATED BY SHAKING AT $50{ }^{\circ} \mathrm{C}$ FOR 10 MIN THEN ALLOWED TO COOL TO $25^{\circ} \mathrm{C}$. THE COOLED MIXTURE WAS EXTRACTED WITH $t$-BUOME $(4 \times 1.0 \mathrm{ML})$, AND THE ORGANIC LAYERS WERE COMBINED, DRIED OVER $\mathrm{NA}_{2} \mathrm{SO}_{4}$, AND CONCENTRATED UNDER REDUCED PRESSURE. THE RESIDUE WAS PURIFIED BY CHROMATOGRAPHY (SILICA GEL, HEXANE) TO GIVE 4-[(1E)3-PHENYLPROP-1-EN-1-YL]BIPHENYL (4aa; 5.4 MG, 0.020 MMOL, 59\%) AS A WHITE SOLID.

4.4.1. 4-[(1E)-3-Phenylprop-1-en-1-yl]biphenyl (4aa). White solid; mp $9091{ }^{\circ} \mathrm{C} ;{ }^{1} \mathrm{H}$ NMR $\left(396 \mathrm{MHz}, \mathrm{CDCl}_{3}\right) \delta 7.60752(\mathrm{~m}$, $4 \mathrm{H}, \mathrm{ArH}), 7.45 \tilde{7} 41(\mathrm{~m}, 4 \mathrm{H}, \mathrm{ArH}), 7.35 \tilde{7} 31(\mathrm{~m}, 2 \mathrm{H}, \mathrm{ArH}), 7.27 \tilde{7} 21$ $(\mathrm{m}, 4 \mathrm{H}, \mathrm{ArH}), 6.50\left(\mathrm{~d}, J=15.6 \mathrm{~Hz}, 1 \mathrm{H},-\mathrm{CH}=\mathrm{CHCH}_{2}-\right), 6.41$ $\left(\mathrm{dt}, J=15.6,6.5 \mathrm{~Hz}, 1 \mathrm{H},-\mathrm{CH}=\mathrm{CHCH}_{2}-\right), 3.58(\mathrm{~d}, J=6.5 \mathrm{~Hz}$, $\left.2 \mathrm{H},-\mathrm{CH}=\mathrm{CHCH}_{2}-\right) ;{ }^{13} \mathrm{C} \mathrm{NMR}\left(100 \mathrm{MHz}, \mathrm{CDCl}_{3}\right) \delta 140.7$, $140.1,139.8,136.5,130.6,129.4,128.7,128.7,128.5,127.2(2 \mathrm{C})$, 126.9, 126.5, 126.2, 39.4; IR (ATR): 1600, 1487, 966, 836, 754, 700, 685, $588 \mathrm{~cm}^{-1}$; EI-MS m/z, $270\left[\mathrm{M}^{+}\right]$; Anal. Calcd for $\mathrm{C}_{21} \mathrm{H}_{18}$ : C, 93.29: H, 6.71\%. Found: C, 93.24: H, 6.71\%.

4.4.2. 1,1'-(1E)-Prop-1-ene-1,3-diyldibenzene (4ba) [CAS: 341244-0]. ${ }^{7 \mathrm{~d} 1} \mathrm{H}$ NMR $\left(396 \mathrm{MHz}, \mathrm{CDCl}_{3}\right) \delta 7.37718(\mathrm{~m}, 10 \mathrm{H}, \mathrm{ArH})$, $6.46\left(\mathrm{~d}, J=15.8 \mathrm{~Hz}, 1 \mathrm{H},-\mathrm{CH}=\mathrm{CHCH}_{2}-\right), 6.36(\mathrm{dt}, J=15.8,6.7$ $\left.\mathrm{Hz}, 1 \mathrm{H},-\mathrm{CH}=\mathrm{CHCH}_{2}-\right), 3.55\left(\mathrm{~d}, J=6.7 \mathrm{~Hz}, 2 \mathrm{H},-\mathrm{CH}=\mathrm{CHCH}_{2}-\right.$ ); ${ }^{13} \mathrm{C}$ NMR $\left(100 \mathrm{MHz}, \mathrm{CDCl}_{3}\right) \delta 140.2,137.5,131.1,129.3$, 128.7, 128.5 (2C), 127.2, 126.2, 126.2, 39.4; EI-MS m/z 194 $\left[\mathrm{M}^{+}\right]$.

4.4.3. 1-Methoxy-4-[(1E)-3-phenylprop-1-en-1-yl]benzene (4ca) [CAS: 35856-81-6]. ${ }^{7 \mathrm{~d}} \mathrm{H}$ NMR (396 MHz, $\left.\mathrm{CDCl}_{3}\right) \delta 7.3372(\mathrm{~m}$, $7 \mathrm{H}, \operatorname{ArH}), 6.83(\mathrm{~d}, J=8.7 \mathrm{~Hz}, 2 \mathrm{H}, \operatorname{ArH}), 6.40(\mathrm{~d}, J=15.7 \mathrm{~Hz}$, $\left.1 \mathrm{H},-\mathrm{CH}=\mathrm{CHCH}_{2}-\right), 6.22(\mathrm{dt}, J=15.7,6.8 \mathrm{~Hz}, 1 \mathrm{H},-$ $\left.\mathrm{CH}=\mathrm{CHCH}_{2}-\right), 3.80\left(\mathrm{~s}, 3 \mathrm{H},-\mathrm{OCH}_{3}\right), 3.53(\mathrm{~d}, J=6.8 \mathrm{~Hz}, 2 \mathrm{H},-$ $\left.\mathrm{CH}=\mathrm{CHCH}_{2}-\right) ;{ }^{13} \mathrm{C}$ NMR $\left(100 \mathrm{MHz} \mathrm{CDCl}_{3}\right) \delta 158.8,140.4$, 130.4, 130.3, 128.6, 128.4, 127.2, 127.0, 126.1, 113.9, 55.3, 39.3; EI-MS $m / z 224\left[\mathrm{M}^{+}\right]$.

4.4.4. 1-Methyl-4-[(1E)-3-phenylprop-1-en-1-yl]benzene (4da) [CAS: 134539-87-0]. ${ }^{7 \mathrm{~d}} \mathrm{H}$ NMR (396 MHz, $\left.\mathrm{CDCl}_{3}\right) \delta 7.35719(\mathrm{~m}$, $7 \mathrm{H}, \operatorname{ArH}), 7.10(\mathrm{~d}, J=8.3 \mathrm{~Hz}, 2 \mathrm{H}, \operatorname{ArH}), 6.43(\mathrm{~d}, J=15.4 \mathrm{~Hz}$, $\left.1 \mathrm{H},-\mathrm{CH}=\mathrm{CHCH}_{2}-\right), 6.30 \quad(\mathrm{dt}, J=15.4,7.0 \mathrm{~Hz}, 1 \mathrm{H},-$ $\left.\mathrm{CH}=\mathrm{CHCH}_{2}-\right), 3.54\left(\mathrm{~d}, J=7.0 \mathrm{~Hz}, 2 \mathrm{H},-\mathrm{CH}=\mathrm{CHCH}_{2}-\right), 2.31(\mathrm{~s}$, $\left.3 \mathrm{H}, \mathrm{CH}_{3}\right) ;{ }^{13} \mathrm{C}$ NMR (100 MHz, $\left.\mathrm{CDCl}_{3}\right) \delta 140.3,136.8,134.7$,
$130.9,129.2,128.7,128.4,128.1,126.1,126.0,39.3,21.2$; EIMS $m / z, 208\left[\mathrm{M}^{+}\right]$.

4.4.5. 1-[(1E)-3-Phenylprop-1-en-1-yl]-4-(trifluoromethyl)benzene (4ea) [CAS: 62056-35-3]. ${ }^{12}{ }^{1} \mathrm{NMR}\left(396 \mathrm{MHz}, \mathrm{CDCl}_{3}\right.$ ) $\delta 7.53(\mathrm{~d}, J=8.0 \mathrm{~Hz}, 2 \mathrm{H}, \operatorname{ArH}), 7.43(\mathrm{~d}, J=8.0 \mathrm{~Hz}, 2 \mathrm{H}, \operatorname{ArH})$, $7.35723(\mathrm{~m}, 5 \mathrm{H}, \mathrm{ArH}), 6.48646\left(\mathrm{~m}, 2 \mathrm{H},-\mathrm{CH}=\mathrm{CHCH}_{2}-\right), 3.57(\mathrm{~d}$, $\left.J=3.7 \mathrm{~Hz}, 2 \mathrm{H},-\mathrm{CH}=\mathrm{CHCH}_{2}-\right) ;{ }^{13} \mathrm{C} \mathrm{NMR}\left(100 \mathrm{MHz}, \mathrm{CDCl}_{3}\right) \delta$ 140.9, 139.6, 132.1, 129.8, 128.9 (q, $J=32.4 \mathrm{~Hz}), 128.7,128.6$, 126.4, 126.3, 125.5 (q, $J=3.8 \mathrm{~Hz}), 124.3$ (q, $J=271.8 \mathrm{~Hz}), 39.4$; EI-MS $m / z, 262\left[\mathrm{M}^{+}\right]$.

4.4.6. 1-Methoxy-2-[(1E)-3-phenylprop-1-en-1-yl]benzene (4fa) [CAS: 1246889-00-6]. ${ }^{13} \mathrm{H}$ NMR (396 MHz, CDCl $\left.\mathrm{CD}_{3}\right) \mathrm{d} 76(\mathrm{~d}, J=$ $7.5 \mathrm{~Hz}, 1 \mathrm{H}, \mathrm{ArH}), 7.4674_{1}(\mathrm{~m}, 2 \mathrm{H}, \mathrm{ArH}), 7.377_{16}(\mathrm{~m}, 4 \mathrm{H}, \mathrm{ArH})$, $6.92685(\mathrm{~m}, 2 \mathrm{H}, \mathrm{ArH}), 6.82\left(\mathrm{~d}, J=15.8 \mathrm{~Hz}, 1 \mathrm{H},-\mathrm{CH}=\mathrm{CHCH}_{2}-\right)$, $6.42\left(\mathrm{dt}, J=15.8,7.1 \mathrm{~Hz}, 1 \mathrm{H},-\mathrm{CH}=\mathrm{CHCH}_{2}-\right), 3.85$ (s, 3H, $\left.\mathrm{OCH}_{3}\right), 3.57\left(\mathrm{~d}, J=7.1 \mathrm{~Hz}, 2 \mathrm{H},-\mathrm{CH}=\mathrm{CHCH}_{2}-\right) ;{ }^{13} \mathrm{C} \mathrm{NMR}(100$ $\mathrm{MHz}, \mathrm{CDCl}_{3}$ ) d 156.4, 140.5, 129.7, 128.6, 128.4, 128.1, 126.5, 126.4, 126.0, 125.7, 120.5, 110.7, 55.3, 39.8; EI-MS $m / z 224$ $\left[\mathrm{M}^{+}\right]$.

4.4.7. 2-[(1E)-3-Phenylprop-1-en-1-yl]naphthalene (4ga) [CAS: 5751-32-6]. ${ }^{14}{ }^{1} \mathrm{H}$ NMR (396 MHz, $\left.\mathrm{CDCl}_{3}\right) 87.79774(\mathrm{~m}, 3 \mathrm{H}$, $\operatorname{ArH}), 7.70(\mathrm{~s}, 1 \mathrm{H}, \mathrm{ArH}), 7.58(\mathrm{dd}, J=8.3,1.8 \mathrm{~Hz}, 1 \mathrm{H}, \mathrm{ArH})$, 7.46739 (m, 2H, ArH), $7.29722(\mathrm{~m}, 5 \mathrm{H}, \mathrm{ArH}), 6.62(\mathrm{~d}, J=15.7$ $\left.\mathrm{Hz}, 1 \mathrm{H},-\mathrm{CH}=\mathrm{CHCH}_{2}-\right), 6.49(\mathrm{dt}, J=15.7,6.9 \mathrm{~Hz}, 1 \mathrm{H},-$ $\left.\mathrm{CH}=\mathrm{CHCH}_{2}-\right), 3.61\left(\mathrm{~d}, J=6.9 \mathrm{~Hz}, 2 \mathrm{H},-\mathrm{CH}=\mathrm{CHCH}_{2}-\right) ;{ }^{13} \mathrm{C}$ NMR $\left(100 \mathrm{MHz}, \mathrm{CDCl}_{3}\right) \delta 140.1,134.9,133.6,132.7,131.1$, 129.6 , 128.7, 128.5, 128.1, 127.8, 127.6, 126.2, 126.1, 125.7, 125.5, 123.5, 39.4; EI-MS $m / z 244\left[\mathrm{M}^{+}\right]$.

4.4.8. 2-[(1E)-3-Phenylprop-1-en-1-yl]thiophene (4ha) [CAS: 1403462-93-0]. ${ }^{15}{ }^{1} \mathrm{H}$ NMR $\left(396 \mathrm{MHz}, \mathrm{CDCl}_{3}\right) \delta 7.3373(\mathrm{~m}, 2 \mathrm{H}$, $\mathrm{ArH}), 7.26721(\mathrm{~m}, 3 \mathrm{H}, \mathrm{ArH}), 7.10(\mathrm{~d}, J=4.8 \mathrm{~Hz}, 1 \mathrm{H}$, thiophene $5-\mathrm{H}), 6.93(\mathrm{dd}, J=4.8,3.2 \mathrm{~Hz}, 1 \mathrm{H}$, thiophene $4-\mathrm{H}), 6.89(\mathrm{~d}, J=$ $3.2 \mathrm{~Hz}, 1 \mathrm{H}$, thiophene $3-\mathrm{H}), 6.56(\mathrm{~d}, J=15.6 \mathrm{~Hz}, 1 \mathrm{H},-$ $\left.\mathrm{CH}=\mathrm{CHCH}_{2}-\right), 6.21\left(\mathrm{dt}, J=15.6,6.7 \mathrm{~Hz}, 1 \mathrm{H},-\mathrm{CH}=\mathrm{CHCH}_{2}-\right)$, $3.51\left(\mathrm{~d}, J=6.7 \mathrm{~Hz}, 2 \mathrm{H},-\mathrm{CH}=\mathrm{CHCH}_{2}\right) ;{ }^{13} \mathrm{C} \mathrm{NMR}(100 \mathrm{MHz}$, $\left.\mathrm{CDCl}_{3}\right) \delta 142.6,139.8,129.1,128.7,128.5,127.2,126.2,124.8$, 124.2, 123.5, 39.1; EI-MS $m / z, 200\left[\mathrm{M}^{+}\right]$.

4.4.9. (2E)-Hex-2-en-1-ylbenzene (4ia) [CAS: 78633-31-5]. ${ }^{7 \mathrm{~d}}{ }^{1} \mathrm{H}$ NMR (396 MHz, $\left.\mathrm{CDCl}_{3}\right) \delta 7.31727$ (m, 2H, ArH), $7.20718(\mathrm{~m}$, $3 \mathrm{H}, \mathrm{ArH}), 5.57\left(\mathrm{dt}, J=15.0,5.9 \mathrm{~Hz}, 1 \mathrm{H},-\mathrm{CH}=\mathrm{CHCH}_{2} \mathrm{Ph}\right), 5.50$ $\left(\mathrm{dt}, J=15.4,5.9 \mathrm{~Hz}, 1 \mathrm{H},-\mathrm{CH}=\mathrm{CHCH}_{2} \mathrm{Ph}\right), 3.33(\mathrm{~d}, J=5.9 \mathrm{~Hz}$, $\left.2 \mathrm{H},-\mathrm{CH}=\mathrm{CHCH}_{2} \mathrm{Ph}\right), 2.00 \quad(\mathrm{q}, \quad J=6.7 \mathrm{~Hz}, 2 \mathrm{H},-$ $\mathrm{CH}_{2} \mathrm{CH}=\mathrm{CHCH}_{2} \mathrm{Ph}$ ), 1.40 (sext, $J=7.4 \mathrm{~Hz}, 2 \mathrm{H},-$ $\left.\mathrm{CH}_{2} \mathrm{CH}_{2} \mathrm{CH}=\mathrm{CHCH}_{2} \mathrm{Ph}\right), 0.90\left(\mathrm{t}, J=7.4 \mathrm{~Hz}, 3 \mathrm{H}-\mathrm{CH}_{3}\right) ;{ }^{13} \mathrm{C}$ NMR $\left(100 \mathrm{MHz}, \mathrm{CDCl}_{3}\right) \delta 141.1,131.9,128.9,128.4,128.3$, 125.8, 39.1, 34.6, 22.6, 13.7; EI-MS m/z $160\left[\mathrm{M}^{+}\right]$.

4.4.10. 1-(Cyclohex-2-enyl)benzene (4ja) [CAS: 15232-96-9]. ${ }^{7 \mathrm{~d}}$ ${ }^{1} \mathrm{H}$ NMR $\left(396 \mathrm{MHz}, \mathrm{CDCl}_{3}\right) \delta 7.32728(\mathrm{~m}, 2 \mathrm{H}, \mathrm{ArH}), 7.23718(\mathrm{~m}$, $3 \mathrm{H}, \mathrm{ArH}), 5.91588(\mathrm{~m}, 1 \mathrm{H},-\mathrm{CH}=\mathrm{CH}-\mathrm{CHPh}-), 5.7357(\mathrm{~m}, 1 \mathrm{H},-$ $\mathrm{CH}=\mathrm{CH}-\mathrm{CHPh}-), 3.43338(\mathrm{~m}, 1 \mathrm{H},-\mathrm{CH}=\mathrm{CH}-\mathrm{CHPh}-), 2.11198$ $\left(\mathrm{m}, 3 \mathrm{H},-\left(\mathrm{CH}_{2}\right)_{3}-\mathrm{CHPh}-\right), 1.77172\left(\mathrm{~m}, 1 \mathrm{H},-\left(\mathrm{CH}_{2}\right)_{3}-\mathrm{CHPh}-\right)$, $1.67151\left(\mathrm{~m}, 2 \mathrm{H},-\left(\mathrm{CH}_{2}\right)_{3}-\mathrm{CHPh}-\right) ;{ }^{13} \mathrm{C} \mathrm{NMR}\left(100 \mathrm{MHz}, \mathrm{CDCl}_{3}\right) \delta$ 140.6, 130.1, 128.3, 128.2, 127.7, 125.9, 41.8, 32.6, 25.0, 21.2; EI-MS $m / z, 158\left[\mathrm{M}^{+}\right]$.

4.4.11. 1-Methyl-4-[(2E)-3-phenylprop-2-en-1-yl]benzene (4bb) [CAS: 134539-86-9]. ${ }^{7 \mathrm{~d} 1} \mathrm{H}$ NMR $\left(396 \mathrm{MHz}, \mathrm{CDCl}_{3}\right) \delta 7.35$ (d, $J=$ $7.5 \mathrm{~Hz}, 2 \mathrm{H}, \mathrm{ArH}), 7.29$ (d, J=7.5 Hz, 2H, ArH), 7.21718 (m, 1H, $\mathrm{ArH}), 7.157_{1}(\mathrm{~m}, 4 \mathrm{H}, \mathrm{ArH}), 6.45(\mathrm{~d}, J=15.6 \mathrm{~Hz}, 1 \mathrm{H},-$ $\left.\mathrm{CH}=\mathrm{CHCH}_{2}-\right), 6.34\left(\mathrm{dt}, J=15.6,6.7 \mathrm{~Hz}, 1 \mathrm{H},-\mathrm{CH}=\mathrm{CHCH}_{2}-\right)$, $3.51\left(\mathrm{~d}, J=6.7 \mathrm{~Hz}, 2 \mathrm{H},-\mathrm{CH}=\mathrm{CHCH}_{2}-\right), 2.33\left(\mathrm{~s}, 3 \mathrm{H}, \mathrm{CH}_{3}\right) ;{ }^{13} \mathrm{C}$ NMR $\left(100 \mathrm{MHz}, \mathrm{CDCl}_{3}\right) \delta 137.5,137.0,135.6,130.8,129.5$, 
129.1, 128.52, 128.45, 127.0, 126.1, 38.9, 21.0; EI-MS m/z 208 $\left[\mathrm{M}^{+}\right]$.

4.4.12. 1-Fluoro-4-[(2E)-3-phenylprop-2-en-1-yl]benzene (4bc) [CAS: 485844-19-7]. ${ }^{\text {7d } 1} \mathrm{H}$ NMR (396 MHz, $\left.\mathrm{CDCl}_{3}\right) \delta 7.377_{1} 8(\mathrm{~m}$, $7 \mathrm{H}, \mathrm{ArH}), 6.99(\mathrm{t}, J=8.1 \mathrm{~Hz}, 2 \mathrm{H}, \mathrm{ArH}), 6.44(\mathrm{~d}, J=15.0 \mathrm{~Hz}$, $\left.1 \mathrm{H},-\mathrm{CH}=\mathrm{CHCH}_{2}-\right), 6.32(\mathrm{dt}, J=15.0,6.6 \mathrm{~Hz}, 1 \mathrm{H},-$ $\left.\mathrm{CH}=\mathrm{CHCH}_{2}-\right), 3.52\left(\mathrm{~d}, \mathrm{~J}=6.6 \mathrm{~Hz}, 2 \mathrm{H},-\mathrm{CH}=\mathrm{CHCH}_{2}-\right) ;{ }^{13} \mathrm{C}$ NMR $\left(100 \mathrm{MHz}, \mathrm{CDCl}_{3}\right) \delta 161.5(\mathrm{~d}, J=243.3 \mathrm{~Hz}), 137.3,135.7$ $(\mathrm{d}, J=3.9 \mathrm{~Hz}), 131.2,130.0(\mathrm{~d}, J=7.7 \mathrm{~Hz}), 128.9,128.5,127.2$, 126.1, $115.2(\mathrm{~d}, J=21.0 \mathrm{~Hz}), 38.43$; EI-MS $m / z 212\left[\mathrm{M}^{+}\right]$.

\section{Acknowledgements}

This work was supported by JST-CREST and JST-ACCEL programs.

\section{References and notes}

1. a) Vriezema, D. M.; Aragonès, M. C.; Elemans, J. A. A. W.; Cornelissen, J. L. M.; Rowan, A. E.; Nolte, R. J. M. Chem. Rev. 2005, 105, 1445-1489; b) Raynal, M.; Ballester, P.; Vidal-Ferran, A.; van Leeuwen, P. W. N. M. Chem. Soc. Rev. 2014, 43, 1734-1787.

2. Walde, P.; Umakoshi, H.; Stano, P.; Mavelli, F. Chem. Commun. 2014, 10177-10197.

3. Selected examples of catalytic applications of self-assembled nanoarchitectures in water, see: a) Goedheijt, M. S.; Hanson, B. E.; Reek, J. N. H.; Kamer, P. C. J.; van Leeuwen, P. W. N. M. J. Am. Chem. Soc. 2000, 122, 1650-1657; b) Delaittre, G.; Reynhout, I C.; Cornelissen, J. J. L. M.; Nolte, R. J. M.; Chem. Eur. J. 2009, 15, 12600-12603; (c) Ferreira, M.; Bricout, H.; Azaroual, N.; Gaillard, C.; Landy, D.; Tilloy, S.; Monflier, E. Adv. Synth. Catal. 2010, 352, 1193-1203; d) Patterson, J. P.; Cotanda, P.; Kelley, E. Z.;

Moughton, A. O.; Lu, A.; Epps III, T. H.; O’Reilly, R. K.; Polym. Chem. 2013, 4, 2033-2039; e) Qin, L.; Zhang, L.; Jin, Q.; Zhang,
J.; Han, B.; Liu, M. Angew. Chem. 2013, 125, 7915-7919; Angew. Chem. Int. Ed. 2013, 52, 7761-7765; (f) van Oers, M. C. M.; Abdelmohsen, L. K. E. A.; Rutjes, F. P. J.; van Hest, J. C. M. Chem. Commun. 2014, 50, 4040-4043.

4. a) Hamasaka, G.; Muto, T.; Uozumi, Y. Angew. Chem 2011, 123, 4978-4980; Angew. Chem. Int. Ed. 2011, 50, 4876-4878; b) Hamasaka, G.; Muto, T.; Uozumi, Y. Dalton Trans. 2011, 40, 8859-8868; (c) Hamasaka, G.; Uozumi, Y. Chem. Commun. 2014, $50,14516-14518$.

5. Sakurai, F.; Hamasaka, G.; Uozumi, Y. Dalton Trans. 2015, 44, 7828-7834.

6. For reviews on allylic substitution reactions, see (a) Tsuji, J. Acc. Chem. Res. 1969, 2, 144-152. (b) Trost, B. M. Tetrahedron 1977 33, 2615-2649. (c) Trost, B. M. Angew. Chem. Int. Ed. Engl. 1989, 28, 1173-1192. (d) Trost, B. M. Acc. Chem. Res. 1996, 29, 355364. (e) Trost, B. M.; van Vranken, D. L. Chem. Rev. 1996, 96, 395-422. (f) Trost, B. M.; Crawley, M. L. Chem. Rev. 2003, 103, 2921-2943. (g) Sundararaju, B.; Achard, M.; Bruneau, C. Chem. Soc. Rev. 2012, 41, 4467-4483. (h) Oliver, S.; Evans, P. A. Synthesis, 2013, 45, 3179-3198.

7. Selected examples for palladium-catalyzed allylic arylation of allyl acetates with organoboron reagents in water, see: a) Uozumi, Y.; Danjo, H.; Hayashi, T. J. Org. Chem. 1999, 64, 3384-3388; b) Nájera, C.; Gil-Moltó, J.; Karlström, S.; Adv. Synth. Catal. 2004, 346, 1798-1811; c) Sarkar, S. M.; Uozumi, Y.; Yamada, Y. M. A. Angew. Chem. 2011, 123, 9609-9613; Angew. Chem. Int. Ed. 2011, 50, 9437-9441; d) Yamada, Y. M. A.; Sarkar, S. M.; Uozumi, Y. J. Am. Chem. Soc. 2012, 134, 3190-3198.

8. Pan, D.; Chen, A.; Su, Y.; Zhou, W.; Li, S.; Jia, W.; Xiao, J.; Liu, Q.; Zhang, L.; Jiao, N. Angew. Chem. 2008, 120, 4807-4810; Angew. Chem. Int. Ed. 2008, 47, 4729-4732.

9. Su, Y.; Jiao, N. Org. Lett. 2009, 11, 2980-2983.

10. Pilarski, L. T.; Selander, N.; Böse, D.; Szabó, K. J. Org. Lett. 2009 , 11, 5518-5521.

11. Shintani, R.; Tsutsumi, Y.; Nagaosa, M.; Nishimura, T.; Hayashi, T. J. Am. Chem. Soc. 2009, 131, 13588-13589.

12. Mino, T.; Kogure, T.; Abe, T.; Koizumi, T.; Fujita, T.; Sakamoto, M. Eur. J. Org. Chem. 2013, 1501-1505.

13. Yang, H.: Sun, P.; Zhu, Y.; Yan, H.; Lu, L.; Qu, X.; Li, T.; Mao, P. Chem. Commun. 2012, 48, 7847-7849.

14. Barluenga, J. ; Florentino, L.; Aznar, F.; Valdés, C. Org. Lett. 2013, 13, 510-513.

15. Sekine, M.; Ilies, L.; Nakamura, E. Org. Lett. 2013, 15, 714-717. 


\section{Graphical Abstract}

To create your abstract, type over the instructions in the template box below.

Fonts or abstract dimensions should not be changed or altered.

A vesicular self-assembled amphiphilic

Leave this area blank for abstract info.

palladium NNC-pincer complex-catalyzed

allylic arylation of allyl acetates with sodium

tetraarylborates in water

Go Hamasaka, Fumie Sakurai, Yasuhiro Uozumi

Institute for Molecular Science, Myodaiji, Okazaki 444-8787, Japan

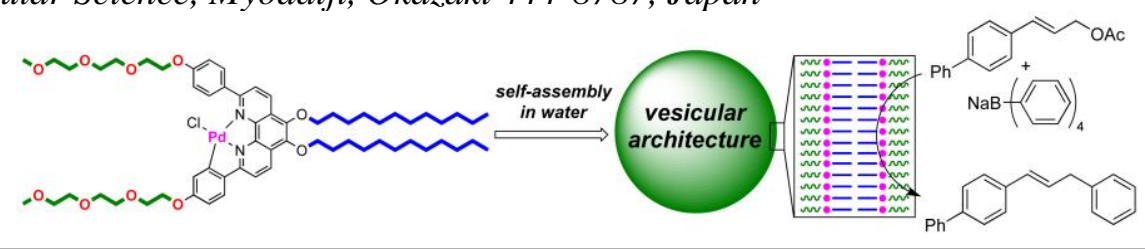

